\title{
Temporal changes in left ventricular longitudinal strain in general population: Clinical correlates and impact on cardiac remodeling
}

\author{
Tatiana Kuznetsova MD, PhD ${ }^{1}$ (D) | Ellen Nijs MSc ${ }^{1}$ | Nicholas Cauwenberghs PhD $^{1}$ | \\ Judita Knez MD, PhD ${ }^{2}$ | Lutgarde Thijs MSc $^{1}$ | Francois Haddad PhD ${ }^{3}$ | Wen-Yi Yang MD, \\ $\mathrm{PhD}^{1} \mid$ Peter L. Kerkhof PhD ${ }^{4}$ Jens-Uwe Voigt MD, PhD ${ }^{5}$ Jan A. Staessen MD, PhD
}

\begin{abstract}
${ }^{1}$ Research Unit Hypertension and Cardiovascular Epidemiology, KU Leuven Department of Cardiovascular Sciences, University of Leuven, Leuven, Belgium

${ }^{2}$ Hypertension Division, Department of Internal Medicine, University Clinical Centre Ljubljana, Ljubljana, Slovenia

${ }^{3}$ Stanford Cardiovascular Institute, Stanford University School of Medicine, Stanford, California

${ }^{4}$ Amsterdam Cardiovascular Sciences, VU University Medical Center, Amsterdam, The Netherlands

${ }^{5}$ Division of Cardiology, KU Leuven Department of Cardiovascular Sciences, University of Leuven, Leuven, Belgium
\end{abstract}

\section{Correspondence}

Tatiana Kuznetsova, MD, PhD, Research Unit of Hypertension and Cardiovascular Epidemiology, KU Leuven Department of Cardiovascular Sciences, University of Leuven, Leuven, Belgium.

Email: tatiana.kouznetsova@med.kuleuven. be

Funding information

The European Union (grant HEALTH-F7305507-HoMAGE) and The European Research Council (advanced grant 2011-294713-EPLORE) supported the Studies Coordinating Centre (Leuven, Belgium). The Studies Coordinating Centre also received grants from the Fonds voor Wetenschappelijk Onderzoek Vlaanderen, Belgium (grants G.0880.13; G.0881.13 and $1120916 \mathrm{~N}$ ). JUV holds a personal research mandate of the Fonds voor Wetenschappelijk Onderzoek Vlaanderen.
Background: Recent studies in patients and general population have reported the role of left ventricular (LV) longitudinal strain (LS) as an independent predictor of outcome. However, there are few data on changes in LS over time. We therefore investigated in a general population clinical correlates of temporal changes in LS. We also explored the potential correlation between temporal changes in LV volumes and LS.

Methods and Results: We measured LV end-systolic (ESV) and end-diastolic (EDV) volumes by conventional echocardiography and LS by 2D speckle tracking in 627 participants (mean age 50.6 years, $51.4 \%$ women; $41.3 \%$ hypertensives) at baseline and after 4.7 years. For statistical analysis, we used the absolute values of LS. In stepwise regression, the magnitude of the decrease in all LV LS indexes over time was greater in men than in women $(P<0.0001)$. Higher baseline mean arterial pressure (MAP), a larger longitudinal increase in MAP, and stopping diuretic treatment during follow-up were related to larger decreases in LS indexes. In multivariable-adjusted analysis, we observed an inverse correlation between baseline ESV and LV LS $(P \leq 0.0017)$. Similarly, lower baseline LS and a larger decrease in LS over time were correlated with a lesser longitudinal decrease in ESV $(P \leq 0.0004)$.

Conclusions: A significant decrease in LS over time was associated with male sex, higher baseline MAP, $\triangle \mathrm{MAP}$, and alteration in antihypertensive treatment. We suggested an interaction between a longitudinal decrease in LV deformation and adverse cardiac remodeling, while underscoring the importance of deformation analysis based on LS assessment in patients at risk.

\section{KEYWORDS}

cardiac remodeling, general population, left ventricular strain, longitudinal changes, systolic function 


\section{1 | INTRODUCTION}

The measurement of left ventricular (LV) myocardial deformation (strain) in the longitudinal direction might be a sensitive index to detect early changes in LV systolic function in patients at high risk for cardiovascular outcome. ${ }^{1}$ Recent clinical studies showed the independent prognostic role of global longitudinal strain (LS) in patients with known cardiovascular diseases such as myocardial infarction ${ }^{2}$ or symptomatic heart failure. ${ }^{3,4}$ Few community-based studies revealed that low global LS was associated with the increased risk of various cardiovascular events independent of traditional risk factors. $^{5-7}$ In addition, it was suggested that separate LS analysis of the LV segments might help to better understand the progression of LV systolic dysfunction. ${ }^{8}$

Previous cross-sectional studies have identified that sex, heart rate, and diastolic blood pressure are important determinants of LS measured by tissue Doppler imaging (TDI) or speckle-tracking techniques. ${ }^{9,10}$ On the other hand, serial imaging studies are also needed to clarify the clinical correlates of temporal change in LS. We, therefore, investigated in a general population clinical correlates of longitudinal changes in LS as well as in LV volumetric indexes. In addition, we explored the relationship between changes in LS and LV volume indexes.

\section{2 | METHODS}

\section{1 | Study population}

The Ethics Committee of University of Leuven approved the Flemish Study on Environment, Genes and Health Outcomes (FLEMENGHO) study. From August 1985 until December 2005, we identified a random population sample stratified by sex and age from a geographically defined area in northern Belgium as described previously. ${ }^{6}$ From 2005 to 2009, we invited 1031 former participants for an examination including detailed echocardiography (Figure S1). In this subsample, we obtained written informed consent from 828 participants (participation rate, $80.3 \%$ ). To study the changes in LV LS and volumes, we invited these subjects for a follow-up examination on average 5 years after their first echocardiographic examination. We excluded 147 participants, because they died $(n=25)$, were lost to follow-up ( $n=19$ ), or declined the invitation for the second examination ( $n=103$ ). We additionally excluded 16 participants because of atrial fibrillation at baseline or/and at follow-up $(n=12)$ or the presence of a pacemaker $(n=4)$. Furthermore, we could not reliably determine LV LS in 38 participants, resulting in a total number of 627 subjects statistically analyzed (Figure S1).

\section{2 | Echocardiography}

\subsection{1 | Data acquisition}

A detailed echocardiographic protocol is provided in the online Data S1. Briefly, one experienced physician (T.K.) performed both echocardiographic examinations using a Vivid7 Pro and Vivid E9 (GE Vingmed, Horten, Norway), respectively, interfaced with a 2.5- to 3.5- $\mathrm{MHz}$ phased array probe in accordance with the recent recommendations. ${ }^{11}$ With the subjects in partial left decubitus, the observer obtained images according to the standardized protocol along the parasternal long and short axes and from the apical 4- and 2-chamber long-axis views, together with a simultaneous ECG signal. All recordings were digitally stored for offline analysis.

\subsection{2 | Offline analysis}

One observer (TK) analyzed the echocardiograms blinded to the participants' characteristics. Images were post processed using a workstation running EchoPac software (GE Vingmed). Measurements were averaged over at least three heart cycles for statistical analysis. From the long-axis parasternal view, LV dimensions were measured at enddiastole from the two-dimensionally guided M-mode tracings. Using the standard Simpson method, LV end-diastolic volume (EDV), endsystolic volume (ESV), and ejection fraction (EF) were derived from the apical 4- and 2 chamber views. EDV and ESV were indexed to body surface area (BSA), denoted as EDVi and ESVi. Transmitral Doppler flow signals were used to measure peak early (E) diastolic velocity. From pulsed-wave TDI recordings, we measured the peak systolic (s') and early diastolic (e') velocities of the mitral annular displacement. We calculated the E/e' ratio by dividing transmitral $E$ peak by e'.

\subsection{3 $2 \mathrm{D} \mathrm{LV}$ strain}

Two observers (T.K. and N.C.) derived LV LS using a myocardial speckletracking software package (Q-analysis, GE Vingmed) using default settings as previously described. ${ }^{6}$ The LV endocardial border was traced at the end-systolic frame of the 2D images from the apical 4-chamber view with the manual point-and-click option. The software automatically tracked myocardial speckle motion and divided the region of interest in LV basal, mid, and apical levels. Four-chamber global LS was automatically calculated. We obtained basal-mid and apical LS by averaging the segmental LS of the respective regions. We used absolute values of midwall LS for statistical analysis.

\subsection{4 | Reproducibility}

Complete information on inter-observer reproducibility of LS is provided in the online Data S1.

\section{3 | Other measurements}

A complete description of the anthropometric and blood pressure measurements is provided in the online Data S1.

\section{4 | Statistical methods}

For database management and statistical analyses, we used SAS software, version 9.4 (SAS Institute, Cary, NC). The central tendency 
and the spread of the data are reported as mean \pm SD or as median and $10 \%-90 \%$ percentiles. We compared within-subject changes in means and proportions by a paired $t$ test and McNemar's test, respectively. Statistical significance was a 2-sided significance level below 0.05 . Using stepwise regression, we determined the clinical correlates of LV LS and volume indexes as well as the 4.7-year changes in these indexes. Characteristics considered as covariables in stepwise regression were age, sex, body mass index (BMI), pulse pressure (PP), mean arterial pressure (MAP), heart rate, history of diabetes mellitus, blood glucose, serum creatinine, serum insulin, and coding for type of antihypertensive drug intake. Stepwise models for changes in LV echocardiographic indexes also included baseline LV index value, follow-up duration, changes in the covariables, and three indicator variables coding for antihypertensive drug class

TAB LE 1 Clinical characteristics of 627 participants at baseline and follow-up

\begin{tabular}{|c|c|c|c|c|}
\hline Characteristic & $\begin{array}{l}\text { Visit } 1 \\
\text { (2005-2009) }\end{array}$ & $\begin{array}{l}\text { Visit } 2 \\
\text { (2009-2013) }\end{array}$ & $\Delta$ & $P$-value \\
\hline \multicolumn{5}{|l|}{ Anthropometrics } \\
\hline Age, y & $50.6 \pm 14.6$ & $55.3 \pm 14.5$ & $+4.72 \pm 0.58$ & $<0.0001$ \\
\hline $\begin{array}{l}\text { Body mass index, } \mathrm{kg} / \\
\mathrm{m}^{2}\end{array}$ & $26.4 \pm 4.14$ & $27.1 \pm 4.19$ & $+0.71 \pm 1.85$ & $<0.0001$ \\
\hline $\begin{array}{l}\text { Waist circumference, } \\
\mathrm{cm}\end{array}$ & $89.9 \pm 12.0$ & $95.3 \pm 12.2$ & $+5.36 \pm 7.31$ & $<0.0001$ \\
\hline $\begin{array}{l}\text { Brachial systolic BP, } \\
\mathrm{mm} \mathrm{Hg}\end{array}$ & $128.6 \pm 16.7$ & $132.1 \pm 16.8$ & $+3.52 \pm 13.5$ & $<0.0001$ \\
\hline $\begin{array}{l}\text { Brachial diastolic BP, } \\
\mathrm{mm} \mathrm{Hg}\end{array}$ & $79.8 \pm 9.38$ & $82.2 \pm 9.73$ & $+2.45 \pm 8.63$ & $<0.0001$ \\
\hline $\begin{array}{l}\text { Brachial pulse } \\
\text { pressure, } \mathrm{mm} \mathrm{Hg}\end{array}$ & $48.8 \pm 14.2$ & $49.9 \pm 15.6$ & $+1.07 \pm 11.3$ & 0.017 \\
\hline $\begin{array}{l}\text { Mean arterial } \\
\text { pressure, } \mathrm{mm} \mathrm{Hg}\end{array}$ & $96.0 \pm 10.4$ & $98.8 \pm 10.2$ & $+2.81 \pm 9.08$ & $<0.0001$ \\
\hline Heart rate, bpm & $60.3 \pm 9.35$ & $60.0 \pm 9.63$ & $-0.32 \pm 7.47$ & 0.29 \\
\hline \multicolumn{5}{|l|}{ Questionnaire data } \\
\hline $\begin{array}{l}\text { Current smoking, n } \\
\text { (\%) }\end{array}$ & $121(19.3)$ & $98(15.6)$ & $-3.7 \%$ & $<0.0001$ \\
\hline Drinking alcohol, n (\%) & $259(41.3)$ & $239(38.1)$ & $-3.2 \%$ & 0.072 \\
\hline Hypertensive, n (\%) & $259(41.3)$ & $316(50.4)$ & $+9.1 \%$ & $<0.0001$ \\
\hline $\begin{array}{l}\text { Treated for hyperten- } \\
\text { sion, } \mathrm{n}(\%)\end{array}$ & $154(24.6)$ & $203(32.4)$ & $+7.8 \%$ & $<0.0001$ \\
\hline$\beta$-blockers, $\mathrm{n}(\%)$ & $94(15.0)$ & $107(17.1)$ & $+2.1 \%$ & 0.066 \\
\hline ACE or ARB, n (\%) & $51(8.14)$ & $86(13.72)$ & $+5.6 \%$ & $<0.0001$ \\
\hline $\begin{array}{l}\text { CCB or } \alpha \text {-blockers, } \mathrm{n} \\
\text { (\%) }\end{array}$ & $26(4.20)$ & $55(8.80)$ & $+4.6 \%$ & $<0.0001$ \\
\hline Diuretics, n (\%) & $55(8.77)$ & $69(11.0)$ & $+2.2 \%$ & 0.054 \\
\hline History of CHD, n (\%) & $22(3.51)$ & $44(7.02)$ & $+3.5 \%$ & $<0.0001$ \\
\hline $\begin{array}{l}\text { History of diabetes, } \mathrm{n} \\
\text { (\%) }\end{array}$ & $21(3.35)$ & $48(7.66)$ & $+4.3 \%$ & $<0.0001$ \\
\hline \multicolumn{5}{|l|}{ Biochemical data } \\
\hline $\begin{array}{l}\text { Serum creatinine, } \\
\mu \mathrm{mol} / \mathrm{L}\end{array}$ & $84.1 \pm 16.2$ & $74.7 \pm 23.2$ & $-9.46 \pm 15.1$ & $<0.0001$ \\
\hline $\begin{array}{l}\text { Total cholesterol, } \\
\mathrm{mmol} / \mathrm{L}\end{array}$ & $5.27 \pm 0.95$ & $5.02 \pm 0.95$ & $-0.25 \pm 0.90$ & $<0.0001$ \\
\hline Serum insulin, $\mu \mathrm{mol} / \mathrm{L}$ & $\begin{array}{l}4.72 \\
(2.00-10.0)\end{array}$ & $\begin{array}{l}5.13 \\
(2.00-12.0)\end{array}$ & $\begin{array}{l}+1.09(-0.57 \\
\text { to } 2.03)\end{array}$ & 0.001 \\
\hline
\end{tabular}

Values are mean $( \pm S D)$, number of subjects $(\%)$, or geometric mean $(10 \%-90 \%$ percentile interval). For longitudinal changes $(\Delta)$, values are mean $( \pm S D)$ or percentage change. Hypertension was defined as a brachial BP of at least $140 \mathrm{~mm} \mathrm{Hg}$ systolic or $90 \mathrm{~mm} \mathrm{Hg}$ diastolic or the use of antihypertensive drugs. $A C E=$ angiotensin-converting enzyme; $A R B=$ angiotensin receptor blockers; $\mathrm{BP}=$ blood pressure; $\mathrm{bpm}=$ beats per minute, $\mathrm{BSA}=$ body surface area; $\mathrm{CCB}=$ calcium channel blockers; and $\mathrm{CHD}=$ coronary heart disease. 


\begin{tabular}{|c|c|c|c|c|}
\hline Characteristic & $\begin{array}{l}\text { Visit } 1 \\
(2005-2009)\end{array}$ & $\begin{array}{l}\text { Visit } 2 \\
\text { (2009-2013) }\end{array}$ & $\Delta$ & $P$-value \\
\hline $\begin{array}{l}\text { LA volume index, } \\
\mathrm{mL} / \mathrm{m}^{2}\end{array}$ & $23.0 \pm 6.06$ & $25.8 \pm 6.64$ & $+2.87 \pm 4.18$ & $<0.0001$ \\
\hline \multicolumn{5}{|l|}{ LV structure } \\
\hline $\begin{array}{l}\text { Internal diameter, } \\
\mathrm{cm}\end{array}$ & $5.05 \pm 0.45$ & $5.03 \pm 0.43$ & $-0.026 \pm 0.30$ & 0.030 \\
\hline Septal wall, cm & $0.98 \pm 0.16$ & $1.00 \pm 0.16$ & $+0.030 \pm 0.12$ & $<0.0001$ \\
\hline Posterior wall, cm & $0.89 \pm 0.14$ & $0.94 \pm 0.12$ & $+0.054 \pm 0.11$ & $<0.0001$ \\
\hline $\begin{array}{l}\text { Relative wall } \\
\text { thickness, cm }\end{array}$ & $0.37 \pm 0.06$ & $0.39 \pm 0.05$ & $+0.018 \pm 0.05$ & $<0.0001$ \\
\hline Mass index, $\mathrm{g} / \mathrm{m}^{2}$ & $92.0 \pm 20.8$ & $95.6 \pm 21.2$ & $+3.72 \pm 12.9$ & $<0.0001$ \\
\hline Length, $\mathrm{cm}$ & $8.12 \pm 0.75$ & $8.11 \pm 0.72$ & $-0.014 \pm 0.52$ & 0.50 \\
\hline \multicolumn{5}{|l|}{ LV diastolic function } \\
\hline TDI e' peak, cm/s & $11.5 \pm 3.56$ & $9.81 \pm 3.36$ & $-1.69 \pm 1.56$ & $<0.0001$ \\
\hline E/e' ratio & $7.04 \pm 2.12$ & $7.39 \pm 2.45$ & $+0.35 \pm 1.43$ & $<0.0001$ \\
\hline \multicolumn{5}{|l|}{ 2D LV volumes } \\
\hline $\mathrm{EDV}, \mathrm{mL}$ & $99.9 \pm 25.6$ & $94.7 \pm 25.1$ & $-4.86 \pm 17.2$ & $<0.0001$ \\
\hline EDV index, $\mathrm{mL} / \mathrm{m}^{2}$ & $53.3 \pm 10.8$ & $50.0 \pm 10.4$ & $-3.23 \pm 9.10$ & $<0.0001$ \\
\hline $\mathrm{ESV}, \mathrm{mL}$ & $37.4 \pm 11.7$ & $36.9 \pm 11.6$ & $-0.40 \pm 8.72$ & 0.28 \\
\hline ESV index, $\mathrm{mL} / \mathrm{m}^{2}$ & $20.0 \pm 5.25$ & $19.4 \pm 5.07$ & $-0.48 \pm 4.53$ & 0.014 \\
\hline \multicolumn{5}{|l|}{ LV systolic function } \\
\hline $\begin{array}{l}\text { Ejection fraction, } \\
\%\end{array}$ & $63.5 \pm 6.43$ & $61.2 \pm 6.43$ & $-2.33 \pm 8.21$ & $<0.0001$ \\
\hline TDI s' peak, cm/s & $9.08 \pm 1.41$ & $8.02 \pm 1.30$ & $-1.06 \pm 1.04$ & $<0.0001$ \\
\hline \multicolumn{5}{|l|}{ 4-chamber LS } \\
\hline Global, \% & $19.7 \pm 2.36$ & $19.5 \pm 2.36$ & $-0.20 \pm 2.32$ & 0.034 \\
\hline Basal-mid, \% & $18.5 \pm 2.26$ & $18.6 \pm 2.18$ & $+0.10 \pm 2.15$ & 0.24 \\
\hline Apical, \% & $23.5 \pm 4.23$ & $22.0 \pm 3.79$ & $-1.44 \pm 4.36$ & $<0.0001$ \\
\hline
\end{tabular}

TABLE 2 Echocardiographic characteristics of 627 participants at baseline and follow-up

Values are mean $( \pm S D)$. $2 D$ indicates two-dimensional; $E D V=$ end-diastolic volume; $E S V=$ endsystolic volume; LA = left atrium; LS = longitudinal strain; $L V=$ left ventricle; TDI = tissue Doppler imaging.

intake (starting or stopping treatment between baseline and followup and remaining on treatment). We set the $P$-values for variables to enter the regression models at 0.10 , and we selected the variables with a $P$-value $<0.05$ for future adjustment.

We investigated the cross-sectional and longitudinal associations between LV volumes and LS indexes by use of a mixed model. We expressed the multivariable-adjusted effect sizes in absolute units of the longitudinal change in LV volume index for a 1-SD increase in the LV strain.

\section{RESULTS}

\section{1 | Characteristics of participants}

At baseline, mean age of the 627 participants (51.4\% women) was $50.6 \pm 14.6$ years. Median follow-up time was 4.7 years ( 5 th to 95 th percentile, 3.7-5.4 years). Tables 1 and 2 show the clinical and echocardiographic characteristics of the participants by examination phase. Tables S1 and S2 show the baseline and follow-up clinical and echocardiographic characteristics of the study participants by sex.

\section{2 | Cross-sectional correlates of LV LS and volume indexes at baseline and follow-up}

Tables S3 and S4 list the correlates of the LV LS and volume indexes by examination phase. At both visits, LS indexes were significantly lower in men compared to women $(P<0.0001)$. Four-chamber global LS decreased with MAP $(P \leq 0.0030)$, whereas it increased with PP $(P \leq 0.0073$; Table S3). Averaged basal-mid LS was inversely associated with MAP, heart rate, and fasting insulin ( $P \leq 0.0098$ for all). Apical LS was positively correlated with age at both visits $(P \leq 0.0072)$.

As expected, at both visits, men had significantly higher EDVi and ESVi compared to women $(P<0.001)$, whereas EF was lower in men ( $P \leq 0.0057$; Table S4). Apart from EDVi at follow-up, both LV volume indexes decreased with age and heart rate $(P \leq 0.010)$ and were significantly higher in participants with diabetes $(P \leq 0.032)$. 


\section{Panel A}
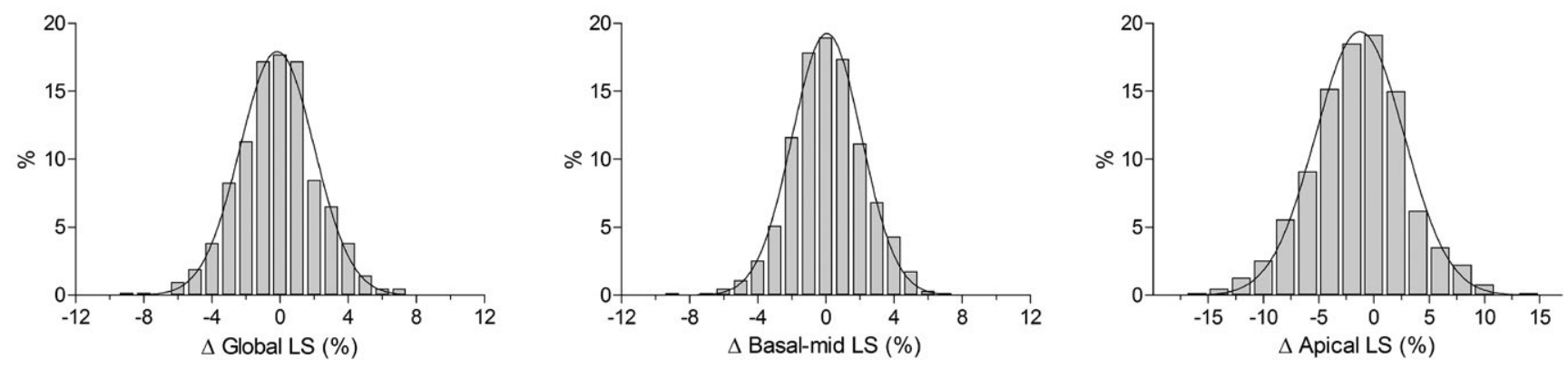

\section{Panel B}
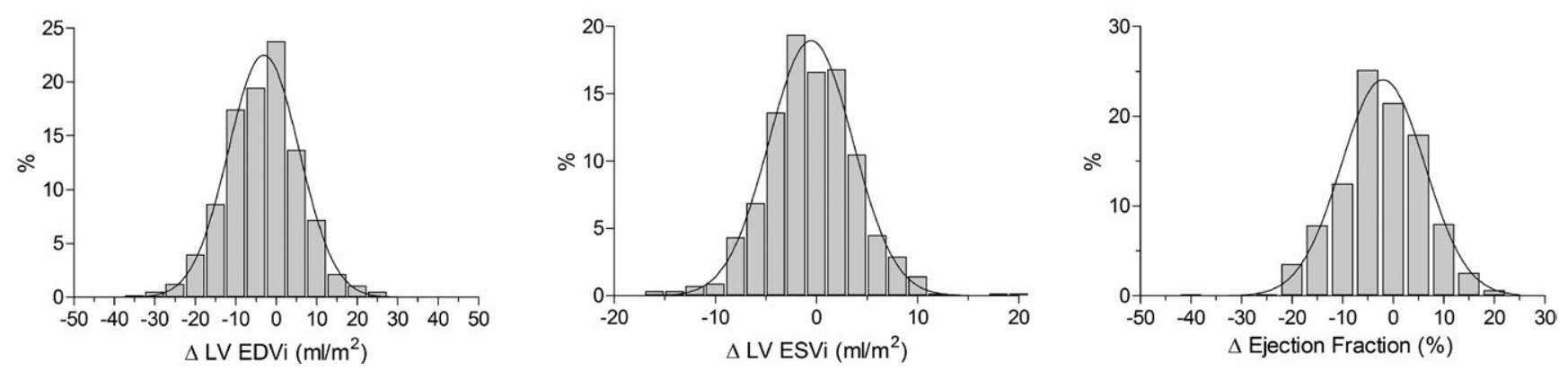

FIGURE 1 The histograms of change in LV 4-chamber LS (panel A) and volumes (panel B) in the entire population. The curve represents the fitted normal plot. EDVi indicates end-diastolic volume index; ESVi = end-systolic volume index; LV = left ventricular; LS = longitudinal strain

\section{3 | Correlates of longitudinal changes in LV strain and volume indexes}

Figure 1 shows the histograms of change over time in the LV LS and volume indexes and $\mathrm{EF}$.

\subsection{1 | LV strain indexes}

During follow-up, 4-chamber global and apical LS tended to decreased significantly by $-0.20 \pm 2.32 \%$ and $-1.44 \pm 4.36 \%$, respectively $(P \leq 0.034)$, whereas basal-mid LS did not show this trend (Table 2). Table 3 presents the correlates of the 4.7-year change in the LS indexes. As expected, we observed significant correlations between changes in any of LV strain indexes with baseline LS index. After adjustment for baseline LS index, the magnitude of decrease in all LS indexes during follow-up was greater in men than in women $(P<0.0001$ for all; Table 3). All LS indexes decreased stronger over time with higher baseline MAP, a longitudinal increase in MAP, and in participants $(n=16)$ who had stopped using diuretics during followup (Table 3). Participants in whom antihypertensive treatment was initiated after the baseline examination $(n=57)$ improved all indexes of LV LS as compared to the rest of the cohort. Of note, basal-mid LS independently and significantly decreased over time with higher baseline serum insulin and with $\Delta$ insulin $(P \leq 0.0022)$.

\subsection{2 | LV volume indexes}

During follow-up, EDVi, ESVi, and EF showed a trend significantly decreased by $-3.23 \pm 9.10 \mathrm{~mL} / \mathrm{m}^{2},-0.48 \pm 4.53 \mathrm{~mL} / \mathrm{m}^{2}$, and $2.33 \pm 8.21 \%$, respectively ( $P \leq 0.014$; Table 2). Table 4 lists the correlates of the 4.7year change in LV volumetric indexes. Similar to LV LS indexes, a longitudinal reduction in all LV volume indexes was significantly correlated with baseline volumes. A stronger longitudinal reduction in ESVi over time was related to higher age $(P=0.0023)$, whereas $\triangle E D V i$ was not associated with age. Hence, EF increased over time with higher age at baseline $(P=0.0004)$. The longitudinal decreases in EDVi and ESVi were less pronounced $(P<0.0001)$ in men than in women. Both EDVi and ESVi reduced stronger over time with higher heart rate at baseline and a greater longitudinal increase in heart rate $(P \leq 0.0013)$. Moreover, we observed a lesser decreases in EDVi and ESVi $(P=0.0047)$ in participants who stopped using diuretics during follow-up.

\subsection{Associations between LV volumes and LV strain}

Table 5 lists the multivariable-adjusted associations between the LV volume and LS indexes at baseline. After full adjustment, baseline ESVi was inversely correlated with all LS indexes $(P \leq 0.0017)$, whereas baseline EDVi was not related to any of these indexes $(P \geq 0.48)$. Consequently, baseline EF was directly related to all baseline LS indexes $(P \leq 0.0073)$.

\subsubsection{Association between temporal changes in LV volumes and LS}

Table 6 shows the multivariable-adjusted estimates for the changes in LV volume indexes associated with a 1-SD decrease in baseline LS index and in LS temporal changes. In multivariable-adjusted analyses, a lesser longitudinal decrease in ESVi was associated with lower baseline 
TABLE 3 Correlates of 4.7-y change in LV LS

\begin{tabular}{|c|c|c|c|c|c|c|c|c|c|}
\hline \multirow[b]{2}{*}{ Variable } & \multicolumn{3}{|l|}{$\Delta$ Global } & \multicolumn{3}{|l|}{ 4-chamber LS } & \multicolumn{3}{|l|}{$\Delta$ Apical } \\
\hline & $\beta \pm \mathrm{SE}$ & $R^{2}(\%)$ & $P$ value & $\beta \pm \mathrm{SE}$ & $R^{2}(\%)$ & $P$ value & $\beta \pm \mathrm{SE}$ & $R^{2}(\%)$ & $P$ value \\
\hline $\begin{array}{l}\Delta, \text { mean }(5-95 \% \\
\mathrm{Cl})\end{array}$ & $\begin{array}{l}-0.20(-3.90 \text { to } \\
3.7)\end{array}$ & 32.2 & & $\begin{array}{l}0.10(-3.24 \text { to } \\
3.75)\end{array}$ & 36.8 & & $\begin{array}{l}-1.44(-8.78 \\
\text { to } 5.90)\end{array}$ & 43.2 & \\
\hline $\begin{array}{l}\text { Years of } \\
\text { follow-up, } \\
+1 \text { y }\end{array}$ & $\ldots$ & & & $\ldots$ & & & $-0.47 \pm 0.23$ & 0.36 & 0.044 \\
\hline \multicolumn{10}{|c|}{ Baseline covariables } \\
\hline $\begin{array}{l}\text { Serum insulin } \\
\text { per doubling }\end{array}$ & $\ldots$ & & & $-3.12 \pm 0.96$ & 0.38 & 0.0013 & $\ldots$ & & \\
\hline $\begin{array}{l}\text { Stop use of } \\
\text { diuretics }\end{array}$ & $-1.28 \pm 0.49$ & 0.90 & 0.0087 & $-1.20 \pm 0.44$ & 0.72 & 0.0063 & $-1.89 \pm 0.84$ & 0.54 & 0.025 \\
\hline $\begin{array}{l}\text { Start } \\
\text { antihyperten- } \\
\text { sive } \\
\text { treatment }\end{array}$ & $0.61 \pm 0.27$ & 0.51 & 0.027 & $0.84 \pm 0.25$ & 1.26 & 0.0007 & $0.88 \pm 0.47$ & 0.32 & 0.064 \\
\hline \multicolumn{10}{|c|}{ Change in covariables } \\
\hline
\end{tabular}

We performed forward stepwise multiple regression to assess the independent correlations between changes in LV longitudinal strain indexes and baseline covariables such as sex, age, body mass index, heart rate, pulse pressure, MAP, history of diabetes mellitus, serum creatinine, total cholesterol, serum insulin, and starting, remaining, or stopping with antihypertensive treatment per drug class. We also included changes in these covariables in the stepwise models. We set the $P$-values for variables to enter the regression models at 0.10 and selected the variables with a $P$-value below 0.05 . Values are mutually adjusted partial regression coefficients \pm SE. LS = longitudinal strain; $L V=$ left ventricular; MAP = mean arterial pressure.

LS $(P \leq 0.0004)$ and with greater longitudinal decrease in LS indexes during follow-up $(P \leq 0.0002$; Table 6). The longitudinal change in EDVi was not related to any of the LS indexes measured at baseline or their changes ( $P \geq 0.08$ for all). Consequently, during follow-up, the decrease in EF was more pronounced in subjects with lower baseline LS and with greater decrease in the LS indexes during follow-up $(P<0.0001$ for all). Figure 2 illustrates the multivariable-adjusted changes in LV volumetric indexes by deciles of relative change in LS.

\section{4 | DISCUSSION}

Because LV LS is widely used in clinical practice for detection of early systolic dysfunction and for patient prognosis, ${ }^{1}$ it is important to better understand the determinants of temporal changes in LV strain indexes during the adult life course. The key finding of this population study is that the decrease in LS over time was more pronounced in men than in women and was associated with higher baseline MAP and $\triangle M A P$ as well as with alteration in antihypertensive treatment over time. In addition, a lesser longitudinal decrease in ESV was related to lower baseline LS and decrease in LS indexes during follow-up. In contrast, EDV did not show any significant correlation with LS indexes or their changes over time.

Left ventricular systolic deformation can be assessed using either magnetic resonance imaging (MRI), TDI, or speckle tracking echocardiography. ${ }^{12}$ Currently, 2D speckle-tracking echocardiography has been used in clinic for the detection of subclinical systolic dysfunction by measuring LV strain. Recent clinical ${ }^{2-4}$ and 
TAB LE 4 Correlates of 4.7-y change in LV volume indexes

\begin{tabular}{|c|c|c|c|c|c|c|c|c|c|}
\hline Variables & \multicolumn{3}{|c|}{$\Delta \operatorname{EDVi}\left(\mathrm{mL} / \mathrm{m}^{2}\right)$} & \multicolumn{3}{|c|}{$\Delta \mathrm{ESVi}\left(\mathrm{mL} / \mathrm{m}^{2}\right)$} & \multicolumn{3}{|l|}{$\Delta \mathrm{EF}(\%)$} \\
\hline$\Delta$, mean $(5-95 \% \mathrm{Cl})$ & $\begin{array}{l}-3.23(-17.9 \\
\text { to } 11.0)\end{array}$ & 33.3 & & $\begin{array}{l}-0.48(-7.89 \\
\text { to } 6.94)\end{array}$ & 32.2 & & $\begin{array}{l}-2.33(-16.1 \\
\text { to } 10.7)\end{array}$ & 44.3 & \\
\hline \multicolumn{10}{|c|}{ Partial regression coefficients $\pm \mathrm{SE}$} \\
\hline $\begin{array}{l}\text { Baseline volume } \\
\text { index, +1 SD }\end{array}$ & $-4.84 \pm 0.31$ & 21.5 & $<0.0001$ & $-2.41 \pm 0.16$ & 20.8 & $<0.0001$ & $-6.89 \pm 0.14$ & 40.5 & $<0.0001$ \\
\hline $\begin{array}{l}\text { Years of } \\
\text { follow-up, + } 1 \text { y }\end{array}$ & $1.24 \pm 0.54$ & & 0.022 & $\ldots$ & & & $\ldots$ & & \\
\hline \multicolumn{10}{|l|}{ Baseline covariables } \\
\hline Age, +10 y & $\ldots$ & & & $-0.35 \pm 0.11$ & 0.86 & 0.0023 & $0.63 \pm 0.17$ & 0.76 & 0.0004 \\
\hline Men & $5.41 \pm 0.72$ & 7.40 & $<0.0001$ & $2.74 \pm 0.36$ & 7.74 & $<0.0001$ & $-1.71 \pm 0.50$ & 1.00 & 0.0006 \\
\hline Start ACE or ARB & $-3.20 \pm 1.37$ & 0.61 & 0.020 & $\ldots$ & & & $-3.28 \pm 1.02$ & 0.85 & 0.0014 \\
\hline \multicolumn{10}{|l|}{ Change in covariables } \\
\hline $\begin{array}{l}\Delta \text { Heart rate, } \\
+10 \text { bpm }\end{array}$ & $-2.48 \pm 0.46$ & 2.31 & $<0.0001$ & $-0.80 \pm 0.23$ & 0.63 & 0.0005 & $\ldots$ & & \\
\hline $\begin{array}{l}\Delta \mathrm{MAP} \\
+10 \mathrm{~mm} \mathrm{Hg}\end{array}$ & $\ldots$ & & & $0.38 \pm 0.18$ & 0.51 & 0.040 & $-0.82 \pm 0.27$ & 0.81 & 0.0031 \\
\hline $\begin{array}{l}\Delta \text { Serum insulin } \\
\text { per doubling }\end{array}$ & $\ldots$ & & & $\ldots$ & & & $-9.32 \pm 3.42$ & 0.42 & 0.0066 \\
\hline
\end{tabular}

We performed forward stepwise multiple regression to assess the independent correlations between changes in LV volumes indexes and baseline covariables such indicated in Table 3. We also included changes in these risk factors in the stepwise models. We set the P-values for variables to enter the regression models at 0.10 and selected the variables with a $P$-value $<0.05$. Values are mutually adjusted partial regression coefficients $\pm S E$. $\mathrm{ACE}$ = angiotensin-converting enzyme; $\mathrm{ARB}=$ angiotensin receptor blockers; $\mathrm{EDVi}=$ end-diastolic volume index; $\mathrm{ESVi}=$ end-systolic volume index; $\mathrm{EF}=$ ejection fraction; $\mathrm{LV}=$ left ventricular; $\mathrm{MAP}=$ mean arterial pressure.

population studies ${ }^{5-7}$ demonstrated the prognostic role of new LV strain indexes. Our previous findings showed that 4-chamber global LS in addition to LV hypertrophy and diastolic dysfunction contributed to risk stratification in 791 FLEMENGHO participants. ${ }^{6}$

Previous cross-sectional studies reported anthropometric and hemodynamic determinants of LS such as BMI, heart rate, and blood pressure. ${ }^{10,13,14}$ It was also shown that men have significantly lower LS compared to women ${ }^{10}$ even in healthy subjects without cardiovascular risk factors. ${ }^{6}$ Similarly, in our cross-sectional analyses, we showed that male sex, heart rate, and MAP are important determinants of LV LS measured at baseline or follow-up. Moreover, in line with previous studies reporting an association between diabetes and alteration in LS, ${ }^{15,16}$ we found that low LV LS indexes at baseline were associated with high fasting insulin level.

In our longitudinal analysis, the same covariables were associated with changes in LV LS indexes over time. Indeed, a decrease in LS indexes during follow-up was more pronounced in men than in women. All LS indexes were also inversely related to baseline MAP and change in MAP over time, which is in line with our previous cross-sectional findings ${ }^{17} \mathrm{~A}$ possible explanation is that an increased MAP leads to increased LV wall stress, particularly on the longitudinally oriented and less curved fibers in the subendocardium, and this impairs myocardial deformation in this direction. ${ }^{18}$ Previous studies showed that deformation in the LV is not uniform and is associated with regional increases in wall stress and modifications of wall curvature. ${ }^{8}$ For instance, in experimental canine model which takes into account LV pressure, regional wall thickness, and meridional and circumferential regional radii of curvature, De Anda et al $^{19}$ reported that regional end-systolic and maximal LV wall stress were heterogeneous and increasing from apex to base $(P<0.001)$. Moreover, regional assessment of wall stress in the LV with magnetic resonance imaging in healthy subjects showed a $55 \pm 13 \%$ increase of the circumferential mean of the peak systolic wall stress in basal segments as compared to apex. ${ }^{20}$ In line with these observations, in our study a higher MAP at baseline and/or during follow-up predicted a stronger decline in basal-mid LS than in apical LS. Furthermore, initiation of antihypertensive treatment during follow-up led to an improvement in LV LS indexes over time, in particular in basal-mid LS. In addition, LV basal segments also seemed more sensitive to insulin resistance, as a higher fasting insulin level at baseline predicted a future decline in basal-mid LS, but not in apical LS. Taken together, effective management of blood 
TAB LE 5 Multivariable-adjusted correlations between LV volumes and strain indexes at baseline

\begin{tabular}{|c|c|c|c|c|c|c|}
\hline Baseline LS index & \multicolumn{2}{|c|}{ Baseline EDVi $\left(\mathrm{mL} / \mathrm{m}^{2}\right)$} & \multicolumn{2}{|l|}{ Baseline ESVi $\left(\mathrm{mL} / \mathrm{m}^{2}\right)$} & \multicolumn{2}{|l|}{ Baseline EF (\%) } \\
\hline \multicolumn{7}{|c|}{ Global LS, decrease by $2.36 \%$} \\
\hline Absolute & $0.28(-1.11,0.52)$ & 0.48 & $1.18(0.78,1.58)$ & $<0.0001$ & $-1.44(-1.96,-0.94)$ & $<0.0001$ \\
\hline \multicolumn{7}{|c|}{ Basal-mid LS, decrease by $2.26 \%$} \\
\hline \multicolumn{7}{|c|}{ Apical LS, decrease by $4.43 \%$} \\
\hline Absolute & $-0.025(-0.76,0.80)$ & 0.95 & $0.89(0.51,1.31)$ & $<0.0001$ & $-1.48(-1.99,-0.97)$ & $<0.0001$ \\
\hline
\end{tabular}

Parameter estimates (95\% confidence interval) are for a 1-SD decrease in the LV strain index at baseline and are expressed in absolute units of the baseline LV volume indexes. Analyses were adjusted for age, sex, heart rate, and history of diabetes mellitus. Analyses of EF were additionally adjusted for treatment of hypertension, treatment with diuretics, and total cholesterol. All covariables were identified based on stepwise regression analyses (see Table S4). EDV indicates end-diastolic volume; ESV = end-systolic volume; EF = ejection fraction; LS = longitudinal strain.

TAB LE 6 Multivariable-adjusted associations between 4.7-y change in LV volumes and LS

\begin{tabular}{|c|c|c|c|c|c|c|}
\hline 4-chamber LS index & \multicolumn{2}{|l|}{$\Delta \mathrm{EDVi}\left(\mathrm{mL} / \mathrm{m}^{2}\right)$} & \multicolumn{2}{|l|}{$\Delta \mathrm{ESVi}\left(\mathrm{mL} / \mathrm{m}^{2}\right)$} & \multicolumn{2}{|l|}{$\Delta \mathrm{EF}(\%)$} \\
\hline $\begin{array}{l}\text { Baseline, decrease } \\
\text { by } 2.36 \%\end{array}$ & $-0.18(-0.60,0.97)$ & 0.65 & $0.97(0.57,1.37)$ & $<0.0001$ & $-2.39(-2.98,-1.81)$ & $<0.0001$ \\
\hline \multicolumn{7}{|l|}{ Basal-mid LS } \\
\hline $\begin{array}{l}\text { Baseline, decrease } \\
\text { by } 2.26 \%\end{array}$ & $-0.24(-0.58,1.06)$ & 0.56 & $0.76(0.34,1.18)$ & 0.0004 & $-1.76(-2.37,-1.14)$ & $<0.0001$ \\
\hline $\begin{array}{l}\Delta, \text { decrease by } \\
2.15 \%\end{array}$ & $-0.68(-0.070,1.43)$ & 0.076 & $0.73(0.35,1.11)$ & 0.0002 & $-1.53(-2.10,-0.96)$ & $<0.0001$ \\
\hline $\begin{array}{l}\Delta, \text { decrease by } \\
4.36 \%\end{array}$ & $-0.19(-0.61,-0.98)$ & 0.64 & $0.93(0.53,1.33)$ & $<0.0001$ & $-1.88(-2.47,-1.29)$ & $<0.0001$ \\
\hline
\end{tabular}

Parameter estimates (95\% confidence interval) are for a 1-SD decrease in LS strain index at baseline and changes herein during follow-up and are expressed in absolute units of the longitudinal change in LV volume indexes. Analyses were adjusted covariables as identified based on stepwise regression analyses (see Table 4). EDVi = end-diastolic volume index; ESVi = end-systolic volume index; EF = ejection fraction; LS = longitudinal strain.

pressure and insulin resistance might thus be important for optimization of LV systolic performance.

In our study, over 4.7 years of follow-up, a small but significant decrease in LS was observed mainly due to decrease in apical deformation. Of notice, in some participants, we observed significant improvement of the LV deformation over time, whereas in others, the LS indexes deteriorated (Figure 1). To our knowledge, only two relatively small studies in patients with a short follow-up period described changes in LS indexes previously. ${ }^{21,22}$ For instance, Kosmala et al ${ }^{21}$ did not find any significant differences in LV longitudinal $(18.7 \%$ vs $18.6 \% ; P=0.52)$ or circumferential $(17.3 \%$ vs $17.6 \%$; $P=0.10)$ strain in 191 hypertensive patients after 1 year of follow-up. On the other hand, in 112 asymptomatic patients with diabetes mellitus and normal ejection fraction, Roos et al $^{22}$ observed a mild but significant decline in LV longitudinal (17.2 \pm 2.3 vs $16.9 \pm 2.7 \%$, $P=0.022)$ and circumferential $(19.7 \pm 4.0$ vs $18.9 \pm 3.8 \%, P<0.001)$ strain after a median follow-up of 2.5 years.

Another key finding of our study is that the changes in LV ESV and EF were related to baseline LV LS indexes as well as to changes in LV strain indexes during follow-up. The interrelation between baseline LV structure and function as well as their temporal changes might help understanding the complex process of LV remodeling associated with age and other cardiovascular risk factors such as hypertension and diabetes.

In line with other longitudinal population studies, ${ }^{23,24}$ LV indexed volumes decreased significantly in our study during 

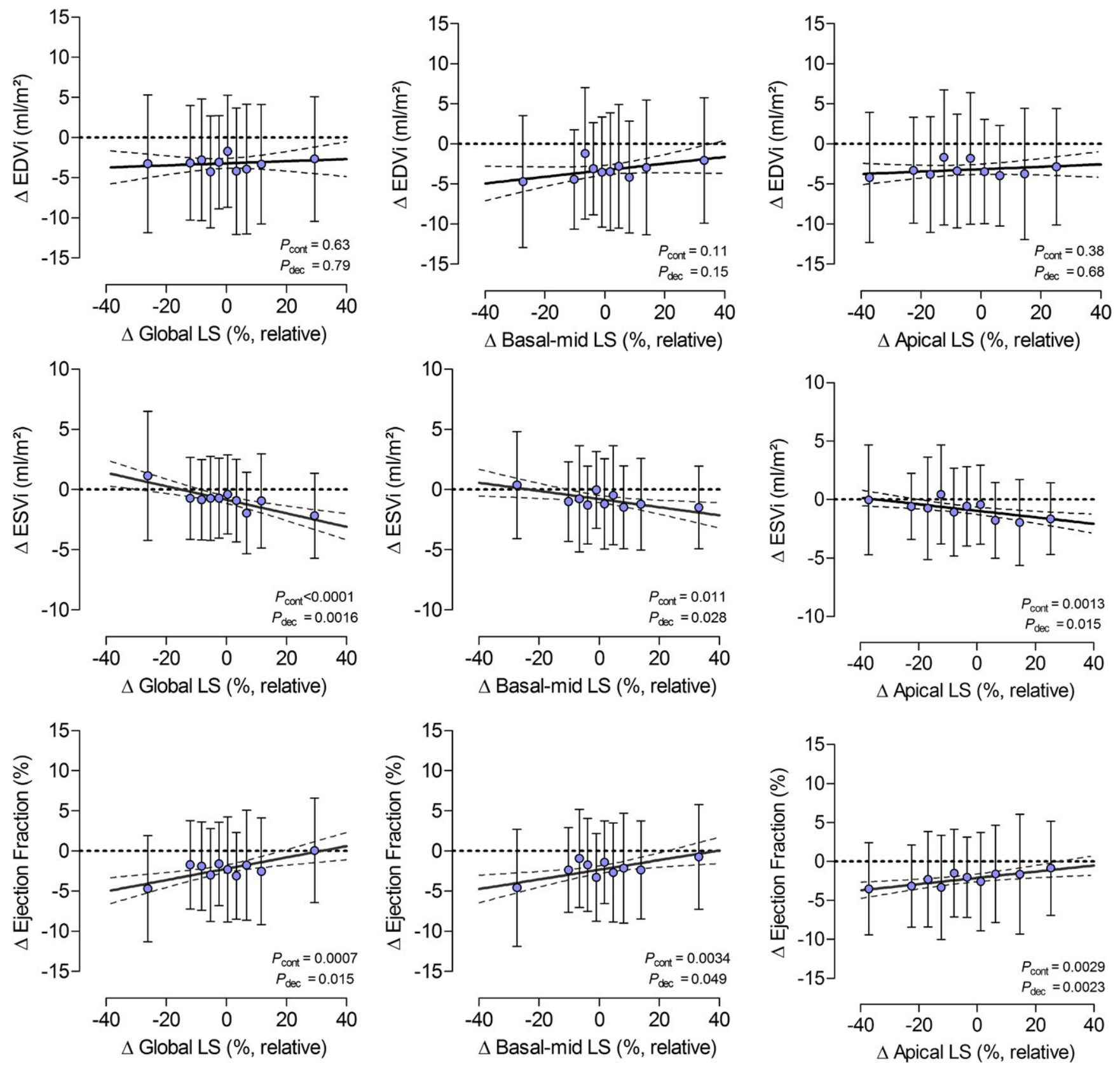

FIGURE 2 Multivariable-adjusted changes in LV volumes during follow-up by deciles of changes in LS. LV volumes were adjusted for important covariables as identified by stepwise regression. $\Delta$ LS indexes are expressed in a relative scale (( $\Delta$ LS/LS baseline) $\times 100 \%)$. Solid and dotted lines represent the regression line and $95 \%$ confidence interval for $\Delta \mathrm{LV}$ volumes vs $\Delta \mathrm{LS}$ indexes on a continuous scale, respectively. $P$-values are for linear trend between $\Delta \mathrm{LV}$ volumes and $\Delta \mathrm{LV} \mathrm{LS}$ on a continuous $\left(P_{\text {cont }}\right)$ or decile scale $\left(P_{\text {dec }}\right)$. EDV $=$ end-diastolic volume; ESV = end-systolic volume; LS = longitudinal strain; LV = left ventricular

4.7 years of follow-up. However, the age-associated LV remodeling may have been affected by sex or other risk factors such as hypertension and diabetes. ${ }^{23,24}$ Indeed, previous studies observed that the presence of these risk factors was associated with less decrease in LV dimensions at older age despite a more pronounced increase in LV wall thickness. ${ }^{23,24}$ Ambale Venkatesh et $\mathrm{al}^{25}$ reported that in men, the presence of replacement fibrosis assessed by late gadolinium enhancement was significantly associated with LV dilatation and decrease in EF during the follow-up period of 10 years. Similarly, we did not find any significant decrease in ESV during follow-up in men compared to women. In 154 asymptomatic patients with diabetes, Ernande et al ${ }^{15}$ reported that altered global LS (<18\%) was independent of other covariables associated with higher ESV at baseline. Moreover, at 3-year follow-up, LV volumes decreased in patients with normal baseline LS, but not in those with low LS. ${ }^{15}$ Thus, the available data suggest that the myocardial deformation analysis might be important in identifying patients at 
high risk of unfavorable cardiac remodeling over the course of life.

\section{1 | Limitations}

Our study has to be interpreted within the context of its potential limitations. First, echocardiography is prone to measurement errors. In the present study, one experienced observer (TK) recorded all echocardiographic images at baseline and follow-up using a standardized imaging protocol. All images were centrally post processed by two experienced observers. Second, we derived LV LS indexes only from the apical 4-chamber view. Thus, the full spectrum of LS among all LV views might be underestimated. This might be particularly important if there is a suspicion of regional myocardial dysfunction due to ischemia or myocardial infarction. Yet, Farsalinos et $a^{26}$ showed a strong correlation between global LS averaged from three long-axes views and global LS from the 4-chamber view $(r=0.92, P<0.001)$. Moreover, we clearly demonstrated the independent prognostic significance of 4-chamber global LS previously. ${ }^{6}$ Third, we did not evaluate myocardial deformation in circumferential and radial direction. However, as indicated in a recent task force, ${ }^{27}$ LV LS indexes appear to be the most robust echocardiographic metric as compared to circumferential and radial strain, and might thus be easily implemented in clinical practice to detect early systolic dysfunction in high risk patients.

\section{5 | CONCLUSION}

In our longitudinal population study, a decrease in LV LS indexes over time was more pronounced in men and was associated with higher baseline MAP and an increase in MAP during follow-up as well as with alterations in antihypertensive treatment over time. In addition, lower baseline LS and a greater decrease in LS indexes during followup correlated with less temporal decrease in ESV, which might be an early sign of adverse LV remodeling. Therefore, our study suggests an interaction between decreased LV systolic deformation and adverse cardiac remodeling, while underscoring the importance of deformation analysis based on LS assessment in patients at risk for the development of symptomatic HF.

\section{ORCID}

Tatiana Kuznetsova iD http://orcid.org/0000-0002-6278-1979

\section{REFERENCES}

1. Smiseth OA, Torp H, Opdahl A, Haugaa KH, Urheim S. Myocardial strain imaging: how useful is it in clinical decision making? Eur Heart J. 2016;37:1196-1207.

2. Ersbøll M, Valeur N, Mogensen UM, et al. Prediction of all-cause mortality and heart failure admissions from global left ventricular longitudinal strain in patients with acute myocardial infarction and preserved left ventricular ejection fraction. J Am Coll Cardiol. 2013;61:2365-2373.
3. Shah AM, Claggett B, Sweitzer NK, et al. Prognostic importance of impaired systolic function in heart failure with preserved ejection fraction and the impact of spironolactone. Circulation. 2015;132:402-414.

4. Sengeløv M, Jørgensen PG, Jensen JS, et al. Global longitudinal strain is a superior predictor of all-cause mortality in heart failure with reduced ejection fraction. JACC Cardiovasc Imaging. 2015;8:1351-1359.

5. Cheng S, McCabe EL, Larson MG, et al. Distinct aspects of left ventricular mechanical function are differentially associated with cardiovascular outcomes and all-cause mortality in the community. J Am Heart Assoc. 2015;4:e002071.

6. Kuznetsova T, Cauwenberghs N, Knez J, et al. Additive prognostic value of left ventricular systolic dysfunction in a population-based cohort. Circ Cardiovasc Imaging. 2016;9:e004661.

7. Biering-Sørensen T, Biering-Sørensen SR, Olsen FJ, et al. Global longitudinal strain by echocardiography predicts long-term risk of cardiovascular morbidity and mortality in a low-risk general population: The Copenhagen City Heart Study. Circ Cardiovasc Imaging. 2017;10:e005521.

8. Choi HF, Rademakers FE, Claus P. Left-ventricular shape determines intramyocardial mechanical heterogeneity. Am J Physiol Heart Circ Physiol. 2011;301:H2351-H2361.

9. Kuznetsova T, Herbots L, Richart $T$, et al. Left ventricular strain and strain rate in a general population. Eur Heart $J$. 2008;29:2014-2023.

10. Cheng S, McCabe EL, Larson MG, et al. Left ventricular mechanical function: clinical correlates, heritability, and association with parental heart failure. Eur J Heart Fail. 2015;17:44-50.

11. Lang RM, Badano LP, Mor-Avi V, et al. Recommendations for cardiac chamber quantification by echocardiography in adults: an update from the American Society of Echocardiography and the European Association of Cardiovascular Imaging. Eur Heart J Cardiovasc Imaging. 2015;16:233-270.

12. Kirkpatrick JN, Vannan MA, Narula J, Lang RM. Echocardiography in heart failure: applications, utility, and new horizons. J Am Coll Cardiol. 2007;50:381-396.

13. Lembo M, Esposito R, Lo ludice F, et al. Impact of pulse pressure on left ventricular global longitudinal strain in normotensive and newly diagnosed, untreated hypertensive patients. J Hypertens. 2016;34:1201-1207.

14. Menting ME, McGhie JS, Koopman LP, et al. Normal myocardial strain values using $2 \mathrm{D}$ speckle tracking echocardiography in healthy adults aged 20 to 72 years. Echocardiography. 2016;33:1665-1675.

15. Ernande L, Bergerot C, Girerd N, et al. Longitudinal myocardial strain alteration is associated with left ventricular remodeling in asymptomatic patients with type 2 diabetes mellitus. J Am Soc Echocardiogr. 2014;27:479-488.

16. Tadic M, Ilic S, Cuspidi C, et al. Left ventricular mechanics in untreated normotensive patients with type 2 diabetes mellitus: a twoand three-dimensional speckle tracking study. Echocardiography. 2015;32:947-955.

17. Sakiewicz W, Kuznetsova T, Kloch-Badelek M, et al. Tissue Doppler indexes of left ventricular systolic function in relation to the pulsatile and steady components of blood pressure in a general population. J Hypertens 2012;30:403-410.

18. Sengupta PP, Narula J. Reclassifying heart failure: predominantly subendocardial, subepicardial, and transmural. Heart Fail Clin. 2008;4:379-382.

19. DeAnda A Jr, Komeda M, Moon MR, et al. Estimation of regional left ventricular wall stresses in intact canine hearts. Am J Physiol. 1998;275:H1879-H1885.

20. Balzer P, Furber A, Delépine S, et al. Regional assessment of wall curvature and wall stress in left ventricle with magnetic resonance imaging. Am J Physiol. 1999;277:H901-H910. 
21. Kosmala W, Przewlocka-Kosmala M, Sharman JE, Schultz MG, Marwick TH. Stability of left ventricular longitudinal and circumferential deformation over time and standard loading conditions. Eur Heart J Cardiovasc Imaging. 2017;18:1001-1007.

22. Roos CJ, Scholte AJ, Kharagjitsingh AV, Bax JJ, Delgado V. Changes in multidirectional LV strain in asymptomatic patients with type 2 diabetes mellitus: a 2-year follow-up study. Eur Heart J Cardiovasc Imaging. 2014;15:41-47.

23. Eng J, McClelland RL, Gomes AS, et al. Adverse left ventricular remodeling and age assessed with cardiac MR imaging: the MultiEthnic Study of Atherosclerosis. Radiology. 2016;278:714-722.

24. Cheng S, Xanthakis V, Sullivan LM, et al. Correlates of echocardiographic indices of cardiac remodeling over the adult life course: longitudinal observations from the Framingham Heart Study. Circulation. 2010;122:570-578.

25. Ambale Venkatesh B, Volpe GJ, Donekal S, et al. Association of longitudinal changes in left ventricular structure and function with myocardial fibrosis: the Multi-Ethnic Study of Atherosclerosis study. Hypertension. 2014;64:508-515.

26. Farsalinos KE, Daraban AM, Ünlü S, Thomas JD, Badano LP, Voigt JU. Head-to-head comparison of global longitudinal strain measurements among nine different vendors: the EACVI/ ASE Inter-Vendor Comparison Study. J Am Soc Echocardiogr. 2015;28:1171-1181.

27. Voigt JU, Pedrizzetti G, Lysyansky P, et al. Definitions for a common standard for 2D speckle tracking echocardiography: consensus document of the EACVI/ASE/Industry Task Force to standardize deformation imaging. J Am Soc Echocardiogr. 2015;28:183-193.

\section{SUPPORTING INFORMATION}

Additional supporting information may be found online in the Supporting Information section at the end of the article.

Data S1. Methods.

Figure S1. Flow chart of participants in the study

Table S1. Clinical characteristics of men and women at baseline and follow-up.

Table S2. Echocardiographic characteristics of men and women at baseline and follow-up.

Table S3. Correlates of LV LS by examination phase.

Table S4. Correlates of LV volumes by examination phase.

How to cite this article: Kuznetsova T, Nijs E, Cauwenberghs $\mathrm{N}$, et al. Temporal changes in left ventricular longitudinal strain in general population: Clinical correlates and impact on cardiac remodeling. Echocardiography. 2018;00:1-11. https:// doi.org/10.1111/echo.14246 